\title{
Pre-treatment Delta Neutrophil Index as a Predictive Factor in Renal Cell Carcinoma
}

\author{
Osman Barut, Mehmet Kutlu Demirkol, Faruk Kucukdurmaz, Tayfun Sahinkanat and Sefa Resim \\ Department of Urology, Faculty of Medicine, Sutcu Imam University, Turkey
}

\begin{abstract}
Objective: To determine the diagnostic value of the pre-treatment delta neutrophil index (DNI) before treatment in patients with renal cell carcinoma (RCC) and to compare this marker with other routine inflammation markers, such as neutrophil-lymphocyte ratio (NLR) and platelet-lymphocyte ratio (PLR).

Study Design: Descriptive study.

Place and Duration of Study: Department of Urology, Kahramanmaras Sutcu Imam University, Turkey, from February 2017 to January 2020.

Methodology: Data of patients who underwent radical nephrectomy for RCC, were evaluated. For comparison, healthy individuals were included in the study as a control group. Demographic data, such as age, gender, routine laboratory tests, DNI, NLR, and PLR levels of the groups were recorded and compared.

Results: There were 73 patients in the RCC group and 71 healthy individuals in the control group. DNI, NLR and PLR levels were significantly higher in the RCC group ( $p<0.001$, each). DNI and NLR were significantly higher in patients with advanced stage (T3-T4) and high grade (G3-G4). In univariate logistic regression analysis hemoglobin $(p=0.023)$, neutrophil $(p<0.001)$, lymphocyte $(p=0.009)$, platelet $(p<0.001)$, DNI $(p<0.001), N L R(p<0.001)$ and PLR $(p<0.001)$ were identified as predictors for RCC. In multivariate logistic regression analysis, DNI and NLR ( $p<0.001$, each) were found to be the predictors of RCC. Cut-off values were $0.45 \%$ for DNI, 1.80 for NLR, and 130.09 for PLR.

Conclusion: DNI is a new inflammatory marker, which is included in complete blood count parameters; and does not require any additional calculation, unlike NLR and PLR. It can be used in the prediction of RCC.
\end{abstract}

Key Words: Renal cell carcinoma, Delta neutrophil index, Neutrophil-lymphocyte ratio, Platelet-lymphocyte ratio.

How to cite this article: Barut O, Demirkol MK, Kucukdurmaz F, Sahinkanat T, Resim S. Pre-treatment Delta Neutrophil Index as a Predictive Factor in Renal Cell Carcinoma. J Coll Physicians Surg Pak 2021; 31(02):156-161.

\section{INTRODUCTION}

Renal cell carcinoma (RCC) constitutes $3-5 \%$ of adult cancers and is the sixth most common cancer in men and 10th in women. ${ }^{1}$ According to the most recent World Health Organization data, more than 140,000 people die each year due to RCC; and the RCC ranks 13th in cancer-related deaths worldwide. ${ }^{2}$ The incidence of RCC rises in parallel with the increase in the number of cases detected incidentally with the widespread use of imaging methods. Most of the lesions detected are small tumors, but distant metastases are observed in $17 \%$ of patients at the time of diagnosis. ${ }^{3}$

Correspondence to: Dr. Osman Barut, Department of Urology, Faculty of Medicine, Sutcu Imam University, Turkey

E-mail: osmanbrt@hotmail.com

Received: December 15, 2020; Revised: January 13, 2021; Accepted: February 08, 2021

DOI: https://doi.org/10.29271/jcpsp.2021.02.156
Inflammation affects every step of tumorigenesis, such as tumor initiation, progression and metastatic spread in RCCs as in all types of cancer. Malignancies activate tumor-associated inflammation by inducing bone marrow. ${ }^{4}$ Therefore, several studies were strated to be conducted to evaluate different inflammatory markers in both diagnosis and prognosis of cancer patients. Among these markers, neutrophil-lymphocyte ratio (NLR) and platelet-lymphocyte ratio (PLR) were found to be associated with cancer-related outcome. ${ }^{5}$ In addition, the delta neutrophil index (DNI), which indicates the number of immature granulocytes (IG) in peripheral blood, has been described in recent studies. ${ }^{6}$ DNI, which is among the parameters of complete blood count, is an easy and inexpensive accessible inflammatory marker that can be measured by automated systems by detecting neutrophil differentiation and nuclear lobularity changes. ${ }^{7}$

On literature review, there was no such study about the clinical usefulness of DNI in renal mass pathologies. Therefore, it was hypothesised that an elevated DNI would be associated with malignant renaltumors.

The current study aims to determine the diagnostic value of the 
pre-treatment DNI level in patients with RCC and compares its level with other routine inflammatory markers, such as NLR and PLR.

\section{METHODOLOGY}

This descriptive case-control study was approved by the Ethics Committee of Sutcu Imam University, Turkey with approval No. $2020 / 11 / 02$. The data of patients who underwent radical nephrectomy due to the diagnosis of localised RCC from February 2017 to January 2020 were analysed. Seventy-three patients, whose hemograms were evaluated within 14 days before nephrectomy, and 71 completely healthy individuals were included in the study. Patients with other organ malignancies, diagnosed with benign kidney tumors, hematological abnormalities, using anticoagulant drugs, and those with active infections, were excluded from the study.

Routine laboratory tests were recorded together with demographic data, such as age and gender of both groups. A specific automated cell analyser (XN 3000; Sysmex Corp., Kobe, Japan) was used to determine the DNI level and other hematological parameters, including neutrophils, platelets, and lymphocytes. NLR and PLR were calculated manually by dividing the neutrophil count by the lymphocyte; and platelet count by the lymphocyte count, respectively. An automated biochemical analyser (Cobas C-702 module, Roche Diagnostics, Basel, Sweeden) was used for biochemical parameters. For the pathological results of nephrectomy specimens, Fuhrman grade system, TNM classification according to 2017 American Joint Committee, and histological subtype according to 2016 World Health Organization, were used.

SPSS version 22.0 (Chicago, IL, USA) software package was used for statistical analysis. Kolmogorov-Smirnov test was used for the compatibility of variables to normal distribution. If the variables were suitable for normal distribution, the independent sample t-test was used; if not, the Mann-Whitney U-test was used. Data are expressed as mean \pm SD or as median and interquartile range (IQR). The comparisons of proportions were performed with the Chi-square test. Predictive factors associated with RCC were determined by univariate and multivariate logistic regression analysis. Cut-off, sensitivity and specificity values of DNI, NLR and PLR for RCC patients were determined, using a receiver operating characteristic $(R O C)$ curve. $A p<0.05$ was setas statistically significant.

\section{RESULTS}

The mean age of patients in the RCC and control groups were $57.21 \pm 14.22$ and $54.38 \pm 8.50$, respectively $(p=0.149)$. The male / female ratio was $1.28: 1$ and $1.63: 1$ in the RCC and control group, respectively $(p=0.479)$.

The median WBC, neutrophil, and platelet count were found to be statistically higher in the RCC group $\left(8.07 \mathrm{~mm}^{3} \mathrm{vs} .6 .20 \mathrm{~mm}^{3}\right.$; $4.8410^{3} / \mu \mathrm{L}$ vs. $3.010^{3} / \mu \mathrm{L} ; 282.010^{3} / \mu \mathrm{L} v s .256 .010^{3} / \mu \mathrm{L}$, respectively; and $p<0.001$, each). In addition, lymphocyte and hemo- globin levels were significantly lower in the RCC group ( $p=$ 0.029 and $p=0.020$, respectively). In biochemical parameters, the median creatine level was significantly higher in the RCC group $(0.90 \mathrm{mg} / \mathrm{dLvs} .0 .80 \mathrm{mg} / \mathrm{dL}$, respectively and $\mathrm{p}=0.011)$.

DNI, NLR, and PLR levels were significantly higher in the RCC group when compared to the control group $(0.80 \%$ vs. $0.30 \%$; 2.48 vs. $1.32 ; 158.29$ vs. 117.37 , and $p<0.001$ each).

In the histopathological evaluation of the surgical specimens of the patients in the RCC group, clear cell carcinoma was reported in $41(56.2 \%)$ patients, papillary type carcinoma in 17 (23.3\%) patients, chromophobe type carcinoma in 10 (13.7\%) patients, and other type carcinomas in $5(6.8 \%)$ patients. Of the 73 patients, who underwent radical nephrectomy, 55 (75.3\%) were in low stage (T1-T2) and 18 (24.7\%) were in advanced stage. In addition, according to the Fuhrman grading system, 56 (76.7\%) patients had low grade (G1-G2) and 17 (23.3\%) patients had high grade (G3-G4) (Table I).

Table Il shows the levels of DNI, NLR and PLR according to pathological parameters. DNI and NLR were significantly higher in advanced stage (T3-T4) tumors, but there was no difference in $\operatorname{PLR}(p<0.001, p=0.002$ and $p=0.096$, respectively). Similarly, according to Fuhrman nuclear grading system, DNI and NLR were significantly higher in high grade (G3-G4) tumors, but no difference was observed in PLR level $(p<0.001, p=0.017$ and $p=0.095$, respectively).

In univariate logistic regression analysis, hemoglobin ( $p=$ $0.023)$, neutrophil $(p<0.001)$, lymphocyte $(p=0.009)$, platelet $(p<0.001)$, DNI value $(p<0.001)$, NLR $(p<0.001)$, and PLR ( $p$ $<0.001$ ) were identified as predictors for RCC. In multivariate logistic regression analysis, DNI value (odds ratio (OR) = $279.47,95 \%$ confidence interval (CI) 25.89-3016.97, $p<0.001$ ) and $\operatorname{NLR}(\mathrm{OR}=8.71,95 \% \mathrm{Cl}=3.16-24.03, \mathrm{p}<0.001)$ were found to be the predictors of RCC (Table III).

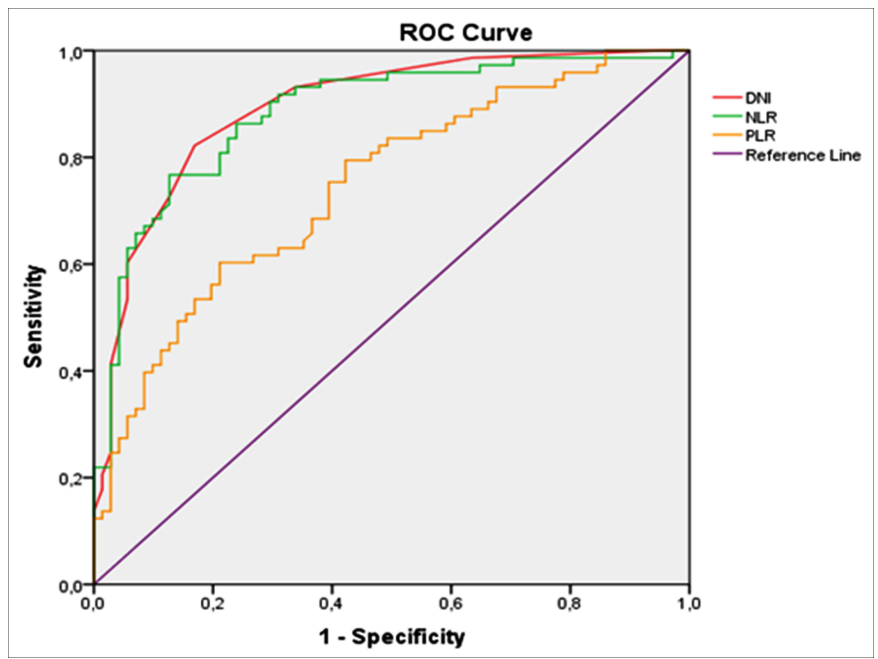

Figure 1: ROC curves of DNI, NLR, and PLR for RCC patients.

The authors performed ROC analysis to determine the sensitivity, specificity; and recommended cut-off values of DNI, NLR and PLR as predictor factors in the diagnosis of RCC. 
Table I: Demographic features of RCC patients and controls.

\begin{tabular}{|c|c|c|c|}
\hline Variables & $\begin{array}{l}\text { RCC patients } \\
(n=73,50.7 \%)\end{array}$ & $\begin{array}{l}\text { Control group } \\
(n=71,49.3 \%)\end{array}$ & $\mathbf{p}$ \\
\hline Age, years & $57.21 \pm 14.22$ & $54.38 \pm 8.50$ & 0.149 \\
\hline \multicolumn{4}{|l|}{ Gender, n (\%) } \\
\hline Male & $41(56.2)$ & $44(62.0)$ & \multirow[b]{2}{*}{0.479} \\
\hline Female & $32(43.8)$ & $27(38.0)$ & \\
\hline \multicolumn{4}{|l|}{ Laboratory tests } \\
\hline WBC $\left(\mathrm{mm}^{3}\right)$ & $8.07(6.60-9.05)$ & $6.20(5.40-6.90)$ & $<0.001$ \\
\hline Neutrophils $\left(10^{3} / \mu \mathrm{L}\right)$ & $4.84(3.79-6.41)$ & $3.0(2.50-3.70)$ & $<0.001$ \\
\hline Lymphocytes $\left(10^{3} / \mu \mathrm{L}\right)$ & $2.02(1.42-2.48)$ & $2.20(1.90-2.50)$ & 0.029 \\
\hline Hemoglobin $(\mathrm{mg} / \mathrm{dL})$ & $13.32 \pm 2.28$ & $14.13 \pm 1.80$ & 0.020 \\
\hline Platelets $\left(10^{3} / \mu \mathrm{L}\right)$ & $282.0(244.0-372.0)$ & $256.0(214.0-286.0)$ & $<0.001$ \\
\hline Glucose (mg/dL) & $100.0(89.50-119.50)$ & $95.0(87.0-124.0)$ & 0.362 \\
\hline $\operatorname{BUN}(\mathrm{mg} / \mathrm{dL})$ & $14.0(12.0-19.0)$ & $13.0(11.0-17.0)$ & 0.080 \\
\hline Creatinine $(\mathrm{mg} / \mathrm{dL})$ & $0.90(0.70-1.07)$ & $0.80(0.60-0.90)$ & 0.011 \\
\hline Sodium (mmol/L) & $141.0(139.5-142.0)$ & $141.0(140.0-143.0)$ & 0.218 \\
\hline Potassium (mmol/L) & $4.50(4.30-4.80)$ & $4.40(4.20-4.70)$ & 0.382 \\
\hline DNI (\%) & $0.80(0.50-1.0)$ & $0.30(0.20-0.40)$ & $<0.001$ \\
\hline NLR & $2.48(1.94-3.18)$ & $1.32(1.12-1.69)$ & $<0.001$ \\
\hline PLR & $158.29(129.26-201.07)$ & $117.37(90.0-141.18)$ & $<0.001$ \\
\hline \multicolumn{4}{|l|}{ Tumor size, $\mathbf{n}(\%)$} \\
\hline$\leq 7 \mathrm{~cm}$ & $65(89.0)$ & & \\
\hline$>7 \mathrm{~cm}$ & $8(11.0)$ & & \\
\hline \multicolumn{4}{|c|}{ Histological subtype, n (\%) } \\
\hline Clear cell & $41(56.2)$ & & \\
\hline Papillary & $17(23.3)$ & & \\
\hline Chromophobe & $10(13.7)$ & & \\
\hline Others & $5(6.8)$ & & \\
\hline \multicolumn{4}{|l|}{ T Stage, n (\%) } \\
\hline T1-T2 & $55(75.3)$ & & \\
\hline T3-T4 & $18(24.7)$ & & \\
\hline \multicolumn{4}{|l|}{ Fuhrman Grade, n (\%) } \\
\hline G1-G2 & $56(76.7)$ & & \\
\hline G3-G4 & $17(23.3)$ & & \\
\hline
\end{tabular}

Table II: DNI, NLR and PLR levels according to pathological parameters.

\begin{tabular}{|l|l|l|l|l|l|l|}
\hline & T1-T2 & T3-T4 & p & G1-G2 & G3-G4 \\
\hline DNI & $0.60(050-0.80$ & $1.40(1.20-1.70)$ & $<0.001$ & $0.60(0.50-0.80)$ & $1.30(1.15-1.80)$ \\
\hline NLR & $2.39(1.77-2.98)$ & $3.17(2.27-5.29)$ & 0.002 & $2.44(1.78-3.05)$ & $2.85(2.19-5.21)$ \\
\hline PLR & $147.24(119.59-203.65)$ & $170.98(149.33-208.42)$ & 0.096 & $149.09(118.84-204.56)$ & $169.44(148.88-197.04)$ \\
\hline \\
DNI = Delta neutrophil index, NLR = Neutrophil-lymphocyte ratio, PLR = Platelet-lymphocyte ratio. 0.095 \\
\hline
\end{tabular}

\begin{tabular}{|c|c|c|c|c|}
\hline \multirow[t]{2}{*}{ Variables } & \multicolumn{2}{|c|}{ Univariate logistic regression } & \multicolumn{2}{|c|}{ Multivariate logistic regression } \\
\hline & OR $(95 \% \mathrm{CI})$ & $\mathbf{p}$ & OR $(95 \% \mathrm{CI})$ & $\mathbf{p}$ \\
\hline Age & $1.02(0.99-1.05)$ & 0.153 & & \\
\hline Gender & $1.27(0.65-2.48)$ & 0.479 & & \\
\hline Creatinine & $2.92(0.91-9.40)$ & 0.073 & & \\
\hline Hemoglobin & $0.82(0.69-0.97)$ & 0.023 & $0.81(0.61-1.08)$ & 0.155 \\
\hline Neutrophils & $3.37(2.20-5.18)$ & $<0.001$ & & \\
\hline Lymphocytes & $0.47(0.27-0.83)$ & 0.009 & & \\
\hline Platelets & $1.01(1.01-1.02)$ & $<0.001$ & & \\
\hline DNI & $530.08(63.58-4418.92)$ & $<0.001$ & $279.47(25.89-3016.97)$ & $<0.001$ \\
\hline NLR & $12.46(5.22-29.71)$ & $<0.001$ & $8.71(3.16-24.03)$ & $<0.001$ \\
\hline PLR & $1.02(1.01-1.03)$ & $<0.001$ & & \\
\hline
\end{tabular}

The cut-off value for DNI was $0.45 \%$ with $82.2 \%$ sensitivity and $83.1 \%$ specificity $(A U C=0.894,95 \% \mathrm{Cl}=0.842-0.946$, $\mathrm{p}<0.001$ ), cut-off value for NLR was 1.80 with $80.8 \%$ sensitivity and $78.9 \%$ specificity $(\mathrm{AUC}=0.884,95 \% \mathrm{Cl}=$ 0.828-0.940, p <0.001), and cut-off value for PLR was
130.09 with $75.3 \%$ sensitivity and $60.6 \%$ specificity (AUC = $0.745,95 \% \mathrm{Cl}=0.666-0.824, \mathrm{p}<0.001)$. ROC curves are shown in Figure 1.

\section{DISCUSSION}


It is known that the inflammatory response can increase neoangiogenesis, cause tumor progression and metastatic spread and local immunosuppression, further increasing genomic instability. ${ }^{8}$ Although there are many studies on NLR and PLR in RCC patients, there are no studies on DNI, which is defined in recent years. The predictive effect of DNI was investigated and found that serum DNI level was higher in pre-treatment RCC patients, compared to the control group. This study shows that the increase in serum DNI level, which can be operated by automated systems and is included in complete blood count parameters, may be a useful infection marker in predicting RCC.

There are many studies demonstrating that systemic inflammatory markers are associated with RCC prediction and prognosis. ${ }^{9}$ NLR is one of the most frequently used markers with its easy to calculate feature. ${ }^{10}$ NLR is characterised by an increase in serum neutrophil levels and / or a decrease in leukocyte count. A high neutrophil count may aid the development and progression of a neoplasia by causing the formation of a favourable tumor microenvironment, in which many growth factors are released. ${ }^{11}$ Ohno et al. reported that 5-year recurrence-free survival rates were $94 \%$ and $78 \%$, in patients with nonmetastatic RCC with a pre-treatment NLR level of $<2.7$ and $\geq 2.7$, respectively. ${ }^{12}$ Viers et al. investigated the relationship between NLR and cancer-specific survival in 827 RCC cases with localised clear cells. They reported that NLR value of 4 or higher negatively affected 5-year overall and cancer-specific survival. ${ }^{13}$ Another study showed that high NLR ( $\geq 1.7$ ) was negatively associated with overall and disease-free survival in nonmetastatic RCC. ${ }^{14}$ In the current study, the authors found that NLR was significantly higher in the RCC group. They calculated a cut-off value of 1.80 for NLR at sensitivity and specificity of $80.8 \%$ and $78.9 \%$, respectively, using the ROC curve.

Tumor cells escape from circulating immune cells and apoptosis, by using platelets as a shield and a link for binding to endothelium via altering expression patterns of adhesion molecules. ${ }^{15}$ Wang et al. showed that high PLR was associated with shorter survival in RCC patients. ${ }^{16}$ In another study, high PLR values were shown to be a marker of advanced disease (T3-4), and the authors reported that high PLR values were associated with a significant decrease in overall survival. ${ }^{17}$ According to a recent meta-analysis, it was reported that disease-free survival or overall survival rates decreased in proportion to the high PLR in patients with RCC. ${ }^{18}$ In this study, it was observed that PLR was higher in RCC patients ( $p$ $<0.001$ ). PLR was found to be an important predictive factor in the univariate analysis, but not in the multivariate analysis.

DNI is a new inflammatory marker, measuring the percentage of immature granulocytes in circulation, measured by new generation automated devices. Studies have revealed that DNI provides early diagnosis in sepsis, shows the severity of the disease, and provides information about the prognosis. ${ }^{19}$ Park et al. reported that DNI value was a more useful early marker than other inflammatory markers in predicting disease severity and prognosis in critically ill patients with sepsis. ${ }^{20}$ DNI values, measured in emergency situations such as acute pyelonephritis, acute cholecystitis and acute appendicitis, have been found to be useful. ${ }^{21-23}$

Bozan et al. compared the preoperative DNI level in patients with nodular goiter and thyroid malignancies. They reported that DNI was higher in the malignant patient group. They found the cut-off value of $0.35 \%$ for the DNI with a sensitivity of $79.2 \%$ and a specificity of $78.9 \%$ for the diagnosis of malignant thyroid diseases. ${ }^{24}$ In the current study, it was observed that the DNI level was significantly higher in the RCC patient group ( $p<0.001)$. DNI and NLR were higher in advanced and high grade RCC patients. However, there was no significant difference in PLR level. Additionally, it was concluded that it was a better inflammatory marker than NLR and PLR with $82.2 \%$ sensitivity and $83.1 \%$ specificity at $0.45 \%$ cut-off value in predicting RCC. While DNI, NLR and PLR were predictive factors for RCC on univariate analysis, only DNI and NLR of these inflammatory markers remain important factors on multivariate analysis. Since there are no studies about the use of preoperative DNI as a predictive factor in patients with RCC, this subject could not be compared with the literature. However, these findings support the hypothesis that the inflammatory response is increased in malignancy without a septic picture.

The main limitations of this study are that it is a retrospective, single-centre study, and the number of malignant patients is low. In addition, the fact that the long-term prognostic effect of DNI cannot be evaluated due to the ongoing postoperative follow-up of the patient group is another limitation. However, the major advantage of this study is its ability to demonstrate the usability of DNI, an easily accessible and inexpensive test for predicting RCC.

\section{CONCLUSION}

DNI is an inflammatory marker that can be useful in RCC prediction, which does not require an additional calculation unlike NLR and PLR, and is included in complete blood count parameters. If the DNI value is $>0.45 \%$ in patients admitted to the urology outpatient clinic because of renal mass, RCC should be suspected and the patients should be evaluated in detail.

\section{ETHICAL APPROVAL:}

All procedures performed in this study, involving human participants, were in accordance with the ethical standards of the Institutional Research Committee and 1964 Helsinki Declaration and its later amendments or comparable ethical standards. This study was approved by Kahramanmaras Sutçu Imam University Medical Ethics Committee, (Ref No. 2020/11/02 Date: 10.06.2020).

\section{PATIENTS' CONSENT:}

Informed consents were obtained from all participants 
included in the study. Additional informed consents were obtained from all participants for whom identifying information is included in this article.

\section{CONFLICT OF INTEREST:}

All authors declared no conflict of interest.

\section{AUTHORS' CONTRIBUTION:}

$\mathrm{OB}$ : Conception of the work, analysis or interpretation of the data for the work, discussion and literature review.

MKD: Data analysis, results, and discussion.

FK: Literature search, analysis, and interpretation of data.

TS: Conception and design, acquisition of data.

SR: Critical revision of the manuscript.

\section{REFERENCES}

1. Siegel RL, Miller KD, Jemal A. Cancer statistics, 2018. CA Cancer J Clin 2018; 68:7-30.

2. Ferlay J, Soerjomataram I, Dikshit R, Eser S, Mathers C, Rebelo M, et al. Cancer incidence and mortality worldwide: Sources, methods and major patterns in Globocan 2012. Int J Cancer 2015; 136(5):E359-86. doi: 10.1002/ijc.29210.

3. Capitanio U, Montorsi F. Renal cancer. Lancet 2016; 387(10021):894-906. doi: 10.1016/S0140-6736(15)00046$\mathrm{X}$.

4. Grivennikov SI, Greten FR, Karin M. Immunity, inflammation, and cancer. Cell 2010; 140(6):883-99. doi.org/10.1016/j.celI.2010.01.025

5. Chrom P, Stec R, Bodnar L, Szczylik C. Incorporating neutrophil-to-lymphocyte ratio and platelet-to-lymphocyte ratio in place of neutrophil count and platelet count Improves prognostic accuracy of the international metastatic renal cell carcinoma database consortium model. Cancer Res Treat 2018; 50(1):103-10. doi: 10.4143/crt.2017.033.

6. Seok Y, Choi JR, Kim J, Kim YK, Lee J, Song J, et al. Delta neutrophil index: A promising diagnostic and prognostic marker for sepsis. Shock 2012; 37(3):242-6. doi: 10.1097/SHK.0b013e3182454acf.

7. Jhun BW, Sim YS, Shin TR, Kim DG. The utility of delta neutrophil index in differetiation of pulmonary tuberculosis from community acquired pneumonia. Sci Rep 2018;_8(1):12343. doi: 10.1038/s41598-018-30967-9.

8. Mantovani A, Allavena P, Sica A, Balkwill F. Cancer-related inflammation. Nature 2008; 454(7203):436-44. doi: 10. 1038/nature07205.

9. Sejima T, Iwamoto H, Morizane S, Hinata N, Yao A, Isoyama $\mathrm{T}$, et al. The significant immunological characteristics of peripheral blood neutrophil-to-lymphocyte ratio and Fas ligand expression incidence in nephrectomized tumor in late recurrence from renal cell carcinoma. Urol Oncol 2013, 31(7):1343-49. doi: 10.1016/j.urolonc.2011.09.008.

10. Keizman D, Ish-Shalom M, Huang P, Eisenberger MA, Pili R, Hammers $H$, et al. The association of pre-treatment neutrophil to lymphocyte ratio with response rate, progression free survival, and overall survival of patients treated with sunitinib for metastatic renal cell carcinoma. Eur J
Cancer 2012; 48(2):202-8. doi: 10.1016/j.ejca.2011.09.001.

11. Kusumanto YH, Dam WA, Hospers GA, Meijer C, Mulder NH. Platelets and granulocytes, in particular the neutrophils, form important compartments for circulating vascular endothelial growth factor. Angiogenesis 2003; 6(4):283-7. doi: 10.1023/B:AGEN.0000029415.62384.ba.

12. Ohno $Y$, Nakashima J, Ohori M, Hatano T, Tachibana M. Pretreatment neutrophil-to-lymphocyte ratio as an independent predictor of recurrence in patients with nonmetastatic renal cell carcinoma. J Urol 2010; 184(3):873-8. doi: 10.1016/j.juro.2010.05.028.

13. Viers BR, Houston Thompson R, Boorjian SA, Lohse CM, Leibovich BC, Tollefson MK. Preoperative neutrophil-lymphocyte ratio predicts death among patients with localized clear cell renal carcinoma undergoing nephrectomy. Urol Oncol 2014; 32(8):1277-84. doi: 10.1016/j.urolonc.2014. 05.014 .

14. Wen RM, Zhang YJ, Ma S, Xu YL, Chen YS, Li HL, et al. Preoperative Neutrophil to Lymphocyte Ratio as a Prognostic Factor in Patients with Non-metastatic Renal Cell Carcinoma. Asian Pac J Cancer Prev 2015; 16(9):3703-8. doi: 10.7314/apjcp.2015.16.9.3703.

15. Kokcu A, Kurtoglu E, Celik H, Tosun M, Malatyalıoglu E, Ozdemir AZ. May the platelet to lymphocyte ratio be a prognostic factor for epithelial ovarian cancer? Asian Pac J Cancer Prev 2014; 15(22):9781-4. doi: 10.7314/apjcp. 2014.15.22.9781.

16. Wang J, Liu Y, Zhang N, Li X, Xin P, Bi J, et al. Prognostic role of pretreatment platelet to lymphocyte ratio in urologic cancer. Oncotarget 2017; 8(41):70874-82. doi: 10. 18632/oncotarget.20147.

17. Peng D, He ZS, Li XS, Tang Q, Zhang L, Yang KW, et al. Prognostic value of inflammatory and nutritional scores in renal cell carcinoma after nephrectomy. Clin Genitourin Cancer 2017; 15(5):582-90. doi: 10.1016/j.clgc.2017.04.001.

18. Wang Z, Peng S, Wang A, Xie H, Guo L, Jiang N, et al. Platelet-lymphocyte ratio acts as an independent predictor of prognosis in patients with renal cell carcinoma. Clin Chim Acta 2018; 480:166-72. doi: 10.1016/j.cca.2018.02.014.

19. Park BH, Kang YA, Park MS, Jung WJ, Lee SH, Lee SK, et al. Delta neutrophil index as an early marker of disease severity in critically ill patients with sepsis. BMC Infect Dis 2011; 11:299. doi: 10.1186/1471-2334-11-299

20. Park JH, Byeon HJ, Lee KH, Lee JW, Kronbichler A, Eisenhut $M$, et al. Delta neutrophil index (DNI) as a novel diagnostic and prognostic marker of infection: A systematic review and meta-analysis. Inflamm Res 2017; 66(10):863-70. doi: 10.1007/s00011-017-1066-y.

21. Barut O, Demirkol MK, Bilecan EB, Sahinkanat T, Resim S. The Delta Neutrophil Index is an Early Predictive Marker of Acute Pyelonephritis in Patients with Ureteral Stone. J Coll Physicians Surg Pak 2020; 30(11):1149-54. doi: 10.29271/jcpsp.2020.11.1149.

22. Lee SJ, Park EJ, Lee KJ, Cha YS. The delta neutrophil index is an early predictive marker of severe acute cholecystitis. Dig Liver Dis 2019; 51(11):1593-8. doi: 10.1016/ j.dld. 2019.03.026.

23. Kim OH, Cha YS, Hwang SO, Jang JY, Choi EH, Kim HI, et al. 
The use of delta neutrophil Index and myeloperoxidase Index for predicting acute complicated appendicitis in children. PLoS One 2016; 11(2):e0148799. doi: 10.1371/journal.pone.0148799.
24. Bozan MB, Yazar FM, Kale IT, Yüzbaşıoğlu MF, Boran ÖF, Azak Bozan A. Delta neutrophil ındex and neutrophil-to-lymphocyte ratio in the differentiation of thyroid malignancy and nodular goiter. World J Surg 2020; 45(2): 507-14. doi: 10.1007/s00268-020-05822-6. 